\title{
Early detection of ovarian cancer
}

\author{
Donna Badgwell and Robert C. Bast Jr.* \\ Department of Experimental Therapeutics, University of Texas M. D. Anderson Cancer Center, Houston, TX, USA
}

\begin{abstract}
Despite advances in therapy, ovarian cancer remains the most deadly of the gynecological cancers. Less than $30 \%$ of women with advanced stage disease survive long-term. When diagnosed in stage I, up to $90 \%$ of patients can be cured with conventional surgery and chemotherapy. At present, only $25 \%$ of ovarian cancers are detected in stage I due, in part, to the absence of specific symptoms and to lack of an effective screening strategy. Early detection of ovarian cancer might significantly improve the overall survival rate of women with ovarian cancer if 1) most cancers are clonal and unifocal, arising in the ovary rather than in the peritoneum, 2) metastatic disease results from progression of clinically detectable stage I lesions, and 3) cancers remain localized for a sufficient interval to permit cost-effective screening. Given the prevalence of ovarian cancer, strategies for early detection must have high sensitivity for early stage disease ( $>75 \%)$, but must have extremely high specificity (99.6\%) to attain a positive predictive value of at least $10 \%$. Transvaginal sonography (TVS), serum markers and a combination of the two modalities have been evaluated for early detection of ovarian cancer. Among the serum markers, CA125 has received the most attention, but lacks the sensitivity or specificity to function alone as a screening test. Greater specificity can be achieved by combining CA125 and TVS and/or by monitoring CA125 over time. Two stage screening strategies promise to be cost effective, where abnormal serum assays prompt TVS to detect lesions that require laparotomy. Accrual has been completed for a 200,000 woman trial in the United Kingdom that will test the ability of a rising CA125 to trigger TVS and subsequent exploratory surgery. Given the heterogeneity of ovarian cancer, it is unlikely that any single marker will be sufficiently sensitive to provide an effective initial screen. Sensitivity of serum assays might be enhanced by utilizing a panel of biomarkers. Candidate biomarkers have been discovered through empirical development of monoclonal antibodies, studies of gene expression, cloning of gene families and proteomic techniques. The development of technologies that measure multiple serum markers simultaneously, linked to the creation of statistical methods that enhance sensitivity without sacrificing specificity hold great promise.
\end{abstract}

Keywords: Ovarian cancer, screening, early detection, biomarkers

\section{Introduction}

Despite advances in cytoreductive surgery and combination chemotherapy, reflected in improved five year survival [1], less than $30 \%$ of women with advanced stage ovarian cancer survive long-term. When ovarian cancer is diagnosed in stage I, up to $90 \%$ of patients can be cured with conventional surgery and chemotherapy. Unfortunately, only $25 \%$ of ovarian cancers are detected in stage I due, in part, to the absence of specific early warning signs. The most commonly reported symptoms prior to diagnosis of ovarian cancer are abdominal

*Corresponding author: Robert C. Bast, Jr., M.D, U.T. M.D. Anderson Cancer Center, Box 355, 1515 Holcombe Boulevard, Houston, TX 77030, USA. Tel.: +1 713792 7743; Fax: +1 713792 7864; E-mail: rbast@mdanderson.org. or pelvic pain, bloating, gastrointestinal distress, and abdominal swelling [93]. Because these symptoms can be indicators of other, more frequently occurring diseases, patients are often misdiagnosed or their diagnosis is delayed [92]. Overall survival and the rate of cure might be significantly improved by the development of an effective screening strategy that could detect disease in its earliest stage prior to clinical presentation.

\section{Attempts to detect early stage ovarian cancer}

\subsection{Biological requirements for early detection}

The ultimate success of any screening strategy for malignant disease depends upon the clinical biology of a cancer. The expectation that screening for ovar- 
ian cancer will impact favorably on survival depends upon several assumptions regarding the biology of the disease. For effective screening: 1) most tumors must arise from single clones of cells within the ovary rather than from multiple foci throughout the abdominal cavity; 2) most metastatic disease should develop by progression from clinically detectable stage I lesions; and 3 ) the length of time that ovarian cancers remain localized to the ovary (Stage I) must be sufficiently long to permit cost-effective screening at practical intervals [7].

In support of the first assumption, our group and others $[35,45,58]$ have shown that most sporadic invasive epithelial ovarian cancers are clonal. When primary ovarian masses and peritoneal metastases have been compared, the same X chromosome is inactivated, the same patterns of loss of heterozygosity are present, and identical p53 mutations are found at both sites in more than $90 \%$ of cases. These studies support a unifocal origin for ovarian cancer.

Screening also depends on the assumption that advanced metastatic disease arises from clinically detectable stage I lesions. Ovarian cancers are thought to arise from epithelial cells that cover the ovarian surface or that line subserosal cysts, but some investigators have proposed that epithelial cancers arise from the rete ovarii [21] or from the epithelial lining of the fallopian tube [43]. It is currently thought that from 4 to 7 genetic alterations may be required to transform normal ovarian epithelial cells into an invasive malignancy. If most metastatic ovarian cancer arises from clinically detectable early stage disease, we would expect to see similar patterns of oncogene activation and tumor suppressor gene loss in all stages of the disease, leading to similar patterns of gene transcription and expression. Results of expression array analysis lend support to the possibility that early stage ovarian cancer is indeed a precursor of late stage disease, at least for the more frequently occurring high grade cancers. Using cDNA microarray analysis, Shridhar, et al, identified genes that were differentially expressed in ovarian cancers when compared to normal ovarian epithelial cell brushings. The same family of genes was expressed in both early and late stage high grade ovarian cancer [83]. These findings are consistent with the possibility that stage I ovarian cancer is, in fact, the precursor of advanced disease.

Even if late stage disease arises from clinically detectable early stage disease, a sufficient interval must exist between the development of potentially detectable early-stage cancer and metastasis to permit screening at practical intervals. To estimate the duration of preclin- ical disease, Skates et al, analyzed CA125 values from 28 patients with ovarian cancer that had been detected during a longitudinal screening trial conducted with 22,000 women in the United Kingdom [34]. Assuming an exponential increase in CA125 with the growth of ovarian cancer, serial CA125 values were fitted using a longitudinal change point model to estimate the interval from tumor inception to clinical detection. The mean estimated duration of preclinical ovarian cancer was $1.9 \pm 0.4$ years. If cancers remained in stage I for half of this interval, annual screening should be effective [90].

\subsection{Epidemiologic requirements for early detection}

Ovarian cancer is neither a common nor a rare disease. Prevalence of epithelial ovarian cancer in the post-menopausal population of the United States or Europe is approximately 1 in 2500 . Therefore, an effective screening strategy must have sensitivity greater than $75 \%$ and specificity greater than $99.6 \%$ to attain a positive predictive value (PPV) of $10 \%$. Although the limit of $10 \%$ for the PPV is arbitrary, most advocates and gynecologic oncologists feel that no more than 10 laparotomies per case of ovarian cancer detected would be acceptable [7]. Several approaches have been evaluated for detecting epithelial ovarian cancer, including TVS, serum markers, and a two-stage screening strategy in which rising serum markers prompt TVS.

\subsection{Approaches to early detection}

\subsubsection{Ultrasonography}

In early studies, transabdominal ultrasonography (TAU) was used to detect ovarian cancer [14]. Improvements in sonographic techniques resulted in the development of transvaginal sonography (TVS). TVS provides a more precise image of the ovary. Subsequent trials of TVS have been conducted in the United Kingdom, United States and Japan [11,78,99]. If the three major studies are considered together, approximately 66,620 women have been screened, prompting 565 operations to detect 45 ovarian cancers, 34 of which were invasive. Approximately 35 of the 45 borderline and invasive cancers $(78 \%)$ were in stage I. Overall, specificity for the major trials is at the margin of that required to achieve a positive predictive value of $10 \%$. One study from the University of Kentucky [99] achieved a positive predictive value (PPV) of 9.9\%, equaling the goal of 10 operations for each case of ovarian cancer detected. Interestingly, the sensitivity of TVS for de- 
tecting stage I ovarian cancer in major trials did not exceed $90 \%$, although this may reflect detection of prevalent disease during an initial screen. Perhaps the major limitation to the widespread use of TVS is the cost of annual screening for the entire postmenopausal population, given the prevalence of ovarian cancer and difficulties in identifying women at increased risk. Modeling of the potential cost and benefit exceeds the limits for other screening tests $[77,97]$.

Women with mutations of BRCA1, BRCA2 or mismatch repair genes are at dramatically increased risk of developing ovarian cancer, but account for only about $10 \%$ of cases. Ninety percent of ovarian cancers are sporadic. Several laboratories are attempting to identify single nucleotide polymorphisms (SNPs) and other more subtle genetic markers that could identify women at moderate risk of developing ovarian cancer, permitting more cost effective screening strategies that would focus on a smaller subset of women at greater than average risk. Although a number of SNPs have been associated with increased or decreased risk, confirmation has proven difficult.

Intuitively, members of ovarian cancer families would seem to be an optimal group to test screening strategies, given the increased incidence of cancer and motivation to participate in screening studies. Biologically, familial ovarian cancer may not, however, be optimal for screening. Women with BRCA1 and BRCA2 mutations often have multifocal disease [43] and primary peritoneal cancer occurs in approximately $5 \%$ of mutation carriers following prophylactic oophorectomy [23]. In microscopic cancers found at prophylactic oophorectomy, p53 mutation is already present in most cases [12]. As p53 mutation has been correlated with metastatic potential [41], it is possible that these small cancers that could not be readily imaged with TVS are already capable of spread. In addition, there are anecdotal reports of women who present with widespread intraperitoneal cancer within 3 months of a normal TVS and CA125. Paradoxically, early detection may be more difficult in this population. In any event, strategies for early detection need to be evaluated in both familial and sporadic groups and it may not be possible to extrapolate from one group to another.

\subsubsection{Serum markers}

Use of serum markers for early detection has largely focused on CA125, a heavily glycosylated highmolecular-weight mucin (MUC 16) [67,107]. Serum CA125 levels are elevated in 50-60\% of patients with early stage ovarian cancer and in $90 \%$ of patients diag- nosed with late stage ovarian cancer [8]. In late stage disease the biomarker is shed not only from cancer cells, but also from activated host mesothelial cells adjacent to peritoneal implants. Overall, significant expression of CA125 is observed in $80 \%$ of ovarian cancers at a tissue level [72], but this varies with histotype. In tissue arrays, CA125 was expressed by $85 \%$ of serous, $68 \%$ of papillary, $65 \%$ of endometrioid, $40 \%$ of clear cell and $36 \%$ of undifferentiated adenocarcinomas, but in only $12 \%$ of mucinous cancers [30].

Aside from limited sensitivity, individual values of CA125 are not sufficiently specific to be used for early detection of ovarian cancer. In postmenopausal women, the CA125 assay exhibits a specificity of $99 \%$, but does not attain the $99.6 \%$ specificity required to achieve a positive predictive value of $10 \%$. In pre-menopausal women, CA125 can be elevated by a variety of benign conditions including menstruation, first trimester pregnancy, endometriosis, adenomyosis and salpingitis. In women of any age, CA125 is elevated by benign ovarian cysts and tumors, uterine fibroids or by inflammation of the pleura, peritoneum or pericardium. In the absence of identifiable benign disease, some women have individual baselines that exceed the usual $35 \mathrm{U} / \mathrm{ml}$ cut off for $99 \%$ specificity $[89,87]$. A further complication is posed by the fact that CA125 can also be elevated by other types of cancer, including carcinomas of the breast and lung $[73,75,86]$. While detection of other cancers might actually be an asset, additional diagnostic studies would be required to evaluate multiple potential primary sites in a woman with a rising CA125. To enhance both specificity and sensitivity, as well as to develop a cost-effective strategy, CA125 has been combined with ultrasonography, both concurrently and sequentially.

\subsubsection{Concurrent combination of CA 125 and transvaginal sonography}

The Prostate, Lung, Colon and Ovary (PLCO) Screening Trial has studied postmenopausal women between 55 and 74 years, randomizing 37,000 to the screening arm of the trial and another 37,000 to participate as non-screened controls [2,28]. For ovarian cancer screening, CA125 levels have been obtained upon entry into the trial and then annually for 5 years. TVS is performed upon entry into the trial and then annually for 3 years. Participants will be followed for a total of 13 years. If CA125 is elevated or a pelvic lesion encountered, patients are referred to their local physicians for management. A serum and plasma bank is being created that will provide valuable serial sam- 
ples from patients that develop ovarian cancer during the course of the study and will also provide samples from normal controls. Thus the PLCO Screening Trial will not only a test the efficacy of CA125 and TVS, but will also provide an important resource for future studies. In a preliminary report from the initial years of the PLCO study, CA125 alone had a PPV of $3.7 \%$ for detection of ovarian cancer, TVS had a PPV of $1 \%$, and both together had a PPV of $23.5 \%$, but $60 \%$ of invasive cancers would not have been detected [13].

\subsubsection{Sequential combination of CA 125 and ultrasonography}

Specificity can be improved by combining CA125 with ultrasonography in a two-stage strategy or by sequential monitoring of CA125 values over time. An early study in the United Kingdom compared the specificity of CA125 alone and in combination with TAU, demonstrating that a combination of CA125 and ultrasound could achieve a specificity of $99.9 \%$ [34]. A more recent and larger study was also conducted in the United Kingdom by Ian Jacobs and his colleagues [36]. Postmenopausal women older than 45 years were randomized to a control group $(10,977)$ or a screened group (10,985). CA125 was measured annually for three years. If CA125 levels were greater than $30 \mathrm{U} / \mathrm{mL}$, TAU was performed. When TAU results were abnormal, surgery was undertaken. Among 10,985 women screened, 29 operations were performed to detect 6 cancers, providing a positive predictive value of $21 \%$. During 7 years of follow-up, 10 more cancers were diagnosed in the screened group. During the same intervals, 21 ovarian cancers were diagnosed in the control group. Median survival in the screened group (73 months) was significantly greater $(p=0.012)$ than in the control group [36].

\subsubsection{Risk of ovarian cancer algorithm}

Patients with benign disease or no disease tend to have stable levels of CA125, even when they are elevated, whereas patients with ovarian cancer generally have progressively rising values. Skates, et al. [91] have analyzed changes in CA125 over time. Their initial analysis considered linear regression of sequential CA125 II values after logarithmic transformation. When the slopes and intercepts were plotted for patients with ovarian cancer and for healthy individuals, the two groups could be distinguished with a specificity of $99.7 \%$ and an apparent sensitivity of $83 \%$, yielding a positive predictive value of $16 \%$. In subsequent studies, Skates, et al, utilized change point analysis to calculate
33,000 sequential serum CA125 values from more than 9000 postmenopausal women at average risk who participated in a screening study coordinated by the Royal London/St Bartholomew's Hospital [89]. The risk of ovarian cancer algorithm (ROCA) using serial CA125 values proved superior to a fixed cutoff of $30 \mathrm{U} / \mathrm{ml}$ for identifying women at increased risk. When specificity was set at $98 \%$, serial values of CA125 achieved a sensitivity of $86 \%$, a significant improvement over the sensitivity of $62 \%$ achieved using the arbitrary cutoff of $30 \mathrm{U} / \mathrm{mL}$. These results led to the development of a computer algorithm that estimates the risk of ovarian cancer being present based on rising CA125 values [55, $56]$.

In a prospective trial, Menon, et al. [55] used CA125 values and the ROCA to classify over 13,000 volunteers older than 50 years of age into normal, intermediate or elevated risk groups. Those at normal risk returned for annual screening. Women with elevated risk were referred immediately for TVS. Women at intermediate risk were asked to repeat CA125 in three months. If CA125 levels and the risk of ovarian cancer were further elevated, TVS was performed; if CA125 was unchanged or decreased participants were asked to return in one year for CA125. This strategy. [74] achieved a specificity of $99.8 \%$ and a positive predictive value of $19 \%$ [55].

Currently, a trial is underway in the United Kingdom that will include 200,000 postmenopausal women who have been randomized to three groups. A control group $(100,000)$ is being followed with conventional pelvic examinations, a second group $(50,000)$ receives annual TVS, and a third group $(50,000)$ has CA125 levels determined at least annually. Based on increases in risk, calculated with the ROC algorithm, patients in the third group are referred for TVS and/or surgery. Women will be screened for 3 years and subsequently followed for up to 7 years. This trial will test the feasibility of screening for ovarian cancer in a postmenopausal population at average risk and will determine whether TVS or a two-stage strategy will impact on survival. Use of the ROC algorithm to prompt TVS may not only improve specificity, but might also improve sensitivity that can be attained with a single value of CA125. CA125 levels are greater than $35 \mathrm{U} / \mathrm{mL}$ in $50 \%$ to $60 \%$ of patients with stage I ovarian cancers at the time of conventional diagnosis. The ROC algorithm could potentially detect rising levels of CA125 and increased risk of disease within the normal range of less than 35 $\mathrm{U} / \mathrm{mL}$, providing sensitivity in excess of $60 \%$. CA125 cannot, however, be detected in tissue sections from 10 
$20 \%$ of ovarian cancers. Consequently, the sensitivity of a CA125-based screening strategy should not exceed $80 \%$. Multiple markers may provide greater sensitivity, provided that specificity is unchanged.

\subsection{Multiple markers may improve sensitivity and specificity}

During the last two decades, a large number of serum tumor markers have been evaluated for their ability to detect early-stage epithelial ovarian cancer. Considering the heterogeneity of ovarian cancers from different patients, it is unlikely that any single marker will be sufficiently sensitive to provide an optimal initial screen. In most early studies [39] the use of multiple markers has improved sensitivity at the expense of a marked decrease in specificity. For example, PetersEngl, et al, evaluated tumor-associated trypsin inhibitor (TATI) and CA125 levels in the serum of patients with ovarian cancer. In this study, the sensitivity and specificity of CA125 were $80 \%$ and $82 \%$ respectively for all stages of disease. The sensitivity and specificity of TATI were $63 \%$ and $72 \%$. When the two markers were considered together, the sensitivity improved to $91 \%$ but the sensitivity decreased to $65 \%$ [69]. Our group has evaluated a Lewis X mucin determinant (OVX1) and the cytokine macrophage colony-stimulating factor (M-CSF) for their ability to detect stage I ovarian cancer and to complement CA125 [101,102]. Among 89 serum samples obtained from patients with stage I ovarian cancer before surgery, CA125 was greater than $35 \mathrm{U} / \mathrm{mL}$ in $69 \%$. A combination of CA125, OVX1, and M-CSF detected $84 \%$ of early-stage cancers and specificity was decreased from $99 \%$ to $84 \%$. From an update of a recent review [6], at least 29 different serum tumor markers have been evaluated in combination with CA125 and reported to increase sensitivity and specificity. Markers have generally been analyzed only two or three at a time. When used in combination with CA125, sensitivity has generally been increased by $5 \%$ to $10 \%$ with multiple markers at the cost of a substantial decrease in specificity. Some studies report more promising results. In another study, researchers showed that sensitivity to early stage disease increased from $45 \%$ for CA125 alone to $70 \%$ when they combined CA125, CA72-4 and M-CSF at a fixed specificity of $98 \%$ [88].

\subsection{Development of mathematical tools to assess multiple markers simultaneously}

The development of reliable analytical tools is a critical component when assessing multiple markers simultaneously. A number of statistical techniques are being developed to allow the complex analysis required to utilize multiple markers simultaneously. These include artificial neural networks, logistic regression, classification tree and mixture discriminant analysis $[88,95]$. Each method has its own strengths and weaknesses. Neural network analysis of multiple marker values has proven superior to CA125 alone in distinguishing benign from malignant pelvic masses [64,113]. Neural network analysis has been compared directly to stepwise regression for distinguishing benign from malignant ovarian tumors. The logistic regression model provided a sensitivity of $66 \%$ and specificity of $78 \%$, whereas a neural network increased both sensitivity to $86 \%$ and specificity to $93 \%$ [64].

Skates et al., investigated the utility of mixed discriminant analysis to combine information from CA125II and three other markers (CA15-3, CA72-4 and macrophage colony stimulating factor (M-CSF)) for distinguishing sera from patients with early stage ovarian cancer from healthy control sera [88]. When logistic regression, classification tree and mixed discriminant analysis were compared for combining multiple markers, the classification tree was the least effective [88]. Logistic regression and mixed discriminant analysis exhibited similar sensitivity for distinguishing women with early stage disease from healthy individuals [88]. At a fixed specificity of $98 \%$, sensitivity was increased from $45 \%$ using CA125II alone to $70 \%$ using a combination of CA125II, CA72-4 and M-CSF.

Multivariate predictive models have been used to combine levels for three proteomic biomarkers in combination with CA125. Sensitivity for early stage disease was increased from $66 \%$ with CA 125 alone to $74 \%$ with the combination of all 4 markers, while specificity was also increased from $52 \%$ to $94 \%$ [114]. In another study, McIntosh et al., evaluated CA125 alone, soluble mesothelin related protein (SMRP) alone and a combination of the two markers. CA125 and SMRP values were transformed using the natural log and then standardized. A combined marker was then estimated using a linear combination of the standardized markers and logistic regression. The combined marker improved sensitivity for ovarian cancer identification without compromising specificity [53]. These studies provide evidence that combining information from 
multiple markers using appropriate statistical methods can improve sensitivity for early stage ovarian cancer, while maintaining specificity.

\subsection{Novel serum biomarkers}

Given mathematical methods for combining multiple biomarker values to improve sensitivity without compromising specificity, there has been an intensive search for additional serum biomarkers that would distinguish patients with early stage or preclinical ovarian cancer from healthy controls. Potential biomarkers have been discovered by multiple methods including the empirical development of monoclonal antibodies, gene expression array analysis, cloning of gene families, and proteomic techniques. Gene expression arrays have proven to be particularly powerful tools for biomarker discovery $[10,37,48,59,84]$, having contributed to the identification of HE4 [31,80], prostasin [59], and osteopontin [37]. Gene expression arrays have also provided useful information concerning the biology of ovarian cancer, molecular changes in different histotypes and signatures that predict prognosis and response to treatment $[26,41,44,51,54,68,81,82]$. For biomarker discovery, it has been essential to show that changes in mRNA expression are reflected at the level of tumor associated protein and that these proteins are shed into body fluid where they can be sampled conveniently.

\subsubsection{HE4}

Overexpression of HE4 in ovarian cancer was first identified in comparative hybridization studies and using serial analysis of gene expression [31,80]. Hellstrom, et al, generated monoclonal antibodies to HE4 and developed an ELISA for the protein. HE4 was detected in the serum of patients with both early and late stage ovarian cancer with sensitivity comparable to CA125 and greater specificity for distinguishing benign from malignant pelvic masses. The initial study included 65 healthy controls, 30 patients with advanced disease and 7 with early stage ovarian cancer. At $100 \%$ specificity, 3 of the 7 (43\%) had elevated HE4.

\subsubsection{Mesothelin}

Mesothelin is another promising candidate for early detection of ovarian cancer. Scholler, et al. were able to detect elevated mesothelin in $77 \%$ of late stage ovarian cancer sera at a specificity of $100 \%$, but no early stage samples were included in this study [79]. In a later study that included some early stage samples, elevated serum mesothelin was detected in $60 \%$ of ovarian cancer sera at $98 \%$ specificity. Importantly, mesothelin was shown to complement serum CA125 and the combination of the two markers detected a greater fraction of ovarian cancers than either marker alone [53]. Our group has recently completed a study in which mesothelin was elevated in $48 \%$ of late stage and $12 \%$ of early stage ovarian cancer serum samples at $95 \%$ specificity. Interestingly, when urine was assayed from the same donors and the values normalized using the glomerular filtration rate, urinary mesothelin was elevated in $42 \%$ of early (stage I-II) cancers and $75 \%$ of late (stage III, IV) cancers. We also found that urinary mesothelin values provide some complementarity to serum CA125 values in the detection of early stage ovarian cancer. CA125 levels were elevated ( $>35$ units $/ \mathrm{ml}$ ) in $75 \%$ of early cancer patient samples. When individual samples were analyzed for serum CA125 and GFR-normalized mesothelin urine levels the detection rate increased to $82 \%$ using the criteria that one or both markers were elevated [2, paper submitted].

\subsubsection{Kallikreins}

Diamandis' group [109] has cloned and cataloged the 15 human kallikrein (hK) genes, some of which have also been shown by expression arrays to be altered in ovarian cancer. The kallikreins are a family of serine proteases that cleave peptides distal to arginine residues (trypsin-like activity) and/or phenylalanine residues (chymotrypsin-like activity). Some kallikreins are overexpressed in ovarian cancers, shed, and activated extracellularly. Kallikreins 6, 10, and 11 have been identified as potential biomarkers for ovarian cancer $[84,108]$. Transcriptional profiling and Northern blot analysis using RNA isolated from normal ovarian epithelium, ovarian cancer cell lines and primary ovarian tumors revealed a markedly upregulated level of kallikrein 10 in some ovarian cancers [84]. Thirtytwo of 35 primary serous ovarian carcinoma samples (91.4\%) expressed elevated levels of hK10 mRNA, when compared to normal ovarian epithelium. Eleven of $15(73.3 \%)$ non-serous epithelial ovarian carcinoma samples and 8 of $10(72.8 \%)$ primary peritoneal carcinomas also overexpressed hK10 at the level of message. Importantly, the serous stage I tissue samples included in the microarray cDNA analysis demonstrated marked overexpression of hK10. At the level of protein, Rosen, et al, utilized tissue arrays to identify potential markers that might complement CA125 expression. Having selected a panel of ovarian cancers that expressed little or no CA125, the expression of 10 other potential markers was assessed using specific antibodies. All 
of the ovarian cancers that lacked CA125 expression were shown to express hK10 and hK6 [72], although these kallikreins were also expressed by several normal tissues. Using an immunoassay for serum hK10 [49], $56 \%$ of ovarian cancer patients had significantly elevated hK10 serum levels compared to healthy women. Similarly, elevated hK11 was found in $70 \%$ of ovarian cancer sera at a specificity of $95 \%$ [18].

\subsubsection{Osteopontin}

Osteopontin was identified as a potential ovarian cancer marker using a cDNA microarray system [100]. Significantly higher levels of osteopontin were found in the serum of patients with epithelial ovarian cancer, including early stage disease, compared to healthy controls [37]. Importantly, osteopontin detection of ovarian cancer is complementary to CA125 [62]. Nakae, et al examined serum from healthy women and women diagnosed with ovarian cancer, including early stage disease. Sensitivity for ovarian cancer was $81 \%$ when osteopontin was used alone compared to $84 \%$ for CA125 alone. A combination of the two markers increased sensitivity to $94 \%$ [65]. Mor, et al have reported that four analytes, including osteopontin, leptin, prolactin and insulin-like growth factor, can achieve a sensitivity of $96 \%$ at a specificity of $94 \%$ in 24 early stage (I/II) ovarian cancers [62]. Studies with larger numbers of early stage cases will be required to judge the utility of the multiple marker combinations. Interestingly, a fragment of osteopontin has also been detected in the urine of ovarian cancer patients [106].

\subsubsection{Prostasin}

Mok et al. [59] demonstrated overexpression of the serine protease prostasin in ovarian cancer cell lines using a cDNA microarray system. Increased prostasin was subsequently detected immunohistochemically in ovarian cancer tissue in a comparison with normal human ovarian surface epithelial cells. Using an ELISA assay, significantly higher prostasin levels were detected in the serum of ovarian cancer patients compared to healthy individuals [59]. Sensitivity for early stage disease has not been defined.

\subsubsection{Macrophage colony stimulating factor $(M-C S F)$ and $O V X 1$}

M-CSF has been found elevated in the serum of $68 \%$ of patients with ovarian cancer at $98 \%$ specificity [101]. M-CSF complements CA125 in the identification of ovarian cancer patients, increasing sensitivity from $64 \%$ to $90 \%$ using the criteria that one or both markers were elevated [101]. A Lewis X mucin determinant (OVX1) also complements M-CSF in distinguishing stage I ovarian cancers from healthy controls [101,102], with an improvement in sensitivity, but with a considerable loss in specificity as described above. A confirmatory study evaluated the combination of CA125II, M-CSF and OVX1 [27]. Sensitivity for stage I disease was improved with the combination of markers $(76 \%)$ compared to CA125 alone $(66 \%)$, but with a substantial loss of specificity. M-CSF has also been shown to improve sensitivity for early stage disease when used in combination with CA72-4 and CA125 [88]. When specificity was fixed at 98\%, a combination of M-CSF, CA72-4 and CA125 achieved a sensitivity of $70 \%$ for stage I ovarian cancer compared to $45 \%$ with CA125 alone [88].

\subsubsection{Lysophosphatidic acid (LPA)}

Elevated levels of LPA have been found in ascites from most ovarian cancer patients [57,103]. LPA levels were initially reported to be elevated in the plasma of $90 \%$ of patients diagnosed with Stage I disease and in $100 \%$ of patients with Stage II, III, and IV at 95\% specificity [104]. In a later study, LPA subspecies were also evaluated. Elevated total LPA levels were detected in the plasma of $80 \%$ of early stage ovarian cancer patients and a combination of the subspecies 16:0 LPA and 20:4 LPA achieved sensitivity for ovarian cancer of $91 \%$ at 96\% specificity [94]. As platelets can contribute some LPA species, measurement of LPA has required plasma rather than serum. As many tissue banks store serum rather than plasma, validation of this biomarker has been difficult. The narrow dynamic range of LPA also requires careful collection of samples and substantial precision in the assay.

\subsubsection{Vascular endothelial growth factor (VEGF)}

VEGF has been detected in ascites, tissue and serum of patients with ovarian cancer [105]. Plasma VEGF levels are significantly higher in ovarian cancer patients than in normal controls or patients with benign disease [50]. In a recent study, Gorelik, et al. demonstrated that a panel of five markers that included VEGF could detect ovarian cancer with a sensitivity of $84 \%$ at $95 \%$ specificity [25]. When Rosen, et al examined the tissue from ovarian cancer patients with low or absent levels of CA125, 81\% of CA125-deficient tissues expressed VEGF, suggesting that VEGF may prove complementary to CA125 [72]. 


\subsubsection{Interleukins}

LabMAP multiplex technology has been utilized to evaluate the usefulness of 24 markers for the early detection of ovarian cancer. Interleukin (IL)- 6 and IL-8 were included as putative markers in this study. When IL-6 and IL- 8 were included in a panel of 5 markers with CA125, a sensitivity for early stage disease of $84 \%$ was achieved at a specificity of $95 \%$ [25]. The same group conducted another study using IL-8, antiIL-8 antibodies and CA125 in early stage ovarian cancer serum samples. When these three markers were combined, a sensitivity of $88 \%$ was achieved at $98 \%$ specificity [47].

\subsubsection{B7-H4}

Using quantitative PCR analysis, B7-H4 was expressed in $100 \%$ of tissue from serous, endometrial and clear cell carcinomas but in only $9 \%$ of mucinous cancers [76]. Importantly, 60\% of Stage I ovarian tissues and $90 \%$ of Stage II tissues stained positive for B7H4 [96]. Elevated levels of B7-H4 were detected in $45 \%$ of early stage cancers at a specificity of $97 \%$ using an ELISA assay [85]. A combination of B7-H4 and CA125 detected a greater fraction of early stage ovarian cancers $(65 \%)$ than either CA125 (52\%) or B7-H4 $(45 \%)$ alone.

\subsubsection{Other candidates identified by gene expression arrays}

Our group used Affymetrix arrays to analyze differences in gene expression of 41,441 known genes and expressed sequence tags between five pools of normal ovarian surface epithelial cells and 42 epithelial ovarian cancers of different stages, grades, and histotypes [48]. Some 86 genes were upregulated at least three-fold over normal epithelium. These genes included claudin 3 (CLDN3), vascular endothelial growth factor (VEGF), Notch homologue 3 (NOTCH3), E2F transcription factor 3 (E2F3), GTPase activating protein (RACGAP1), and hematological and neurological expressed 1 (HN1). The combination of elevated CLDN3 and elevated VEGF distinguished the cancers from normal ovarian surface epithelium. The combination of NOTCH3, E2F3, RACGAP1 and HN1 also distinguished all tumor samples from normal ovarian surface epithelial cells. In the same study, expression of a panel of 11 genes known to encode potential tumor markers [mucin 1, transmembrane (MUC1), mucin 16 (CA125), mesothelin, WAP four-disulfide core domain 2 (HE4), kallikrein 6, kallikrein 10, matrix metalloproteinase 2 , prostasin, osteopontin, tetranectin, and in- hibin] was evaluated using recursive descent partition analysis (RDPA). A combination of HE4, CA125, and MUC1 expression could distinguish tumor from normal specimens [48]. Using immunohistochemistry, a combination of CLDN3, CA125, and MUC1 stained $157(99.4 \%)$ of 158 cancers, and all of the tumors were detected with a combination of CLDN3, CA125, MUC1, and VEGF. These observations are consistent with the possibility that a limited number of markers in combination might identify $>99 \%$ of epithelial ovarian cancers despite the heterogeneity of the disease.

\subsubsection{Proteomic markers}

Biomarkers have been sought by proteomic analysis of sera from ovarian cancer patients and from healthy individuals [63]. Mass spectrometry (MS) uses mass to charge ratios to identify patterns of both known and unknown proteins. Two different approaches have been used. The first attempts to identify differences in patterns that consistently differentiate healthy individuals from cancer patients $[17,24$, 71]. Surface-enhanced laser desorption and ionization (SELDI) or matrix-enhanced laser desorption and ionization (MALDI) have been used to detect novel patterns of low-molecular-weight moieties in serum samples from patients with ovarian cancer. SELDI patterns have been reported to yield $100 \%$ sensitivity and $95 \%$ specificity with a PPV of $94 \%$ for distinguishing ovarian cancer from healthy individuals [70]. This analysis appears, however, to have systematic bias and has proven difficult to confirm [4,5]. Multiple algorithms have been generated with different data sets and it will be important to define which peaks are consistently predictive of early-stage disease. Greater definition has been obtained with matrix assisted laser desorption ionization time of flight (MALDI-TOF) $[16,110]$ than with SELDI, but the possibility of systematic bias in the results has also been raised [4].

A second approach has utilized proteomic patterns as a first step in the discovery of putative biomarkers that can then be analyzed with more conventional techniques $[38,39,46,106,111]$. Once a peak has been identified, the protein is purified and the purified protein is enzymatically digested. The resulting peptides are sequenced and the protein identified. Zhang, et al used SELDI as a first step in the identification of three putative biomarkers for the detection of early stage ovarian cancer. This group showed differential expression of apolipoprotein A1, a truncated form of transthyretin and a cleavage fragment of inter-alphatrypsin inhibitor heavy chain 4 (ITIH4) between serum 
from patients with ovarian cancer and healthy women [114]. They were able to show an increase in sensitivity for early stage ovarian cancer for the three markers and CA125 combined (74\%) when compared to CA125 alone $(65 \%)$ at a fixed specificity of $97 \%$. The value of at least two of these markers was independently confirmed with serum samples from the Mayo Clinic. High sensitivity (79\%) and specificity (94\%) were attained using transthyretin cleavage fragments, apolipoprotein A1, CA125 and age [60].

\subsection{Use of multiplexed assays}

One disadvantage of mass spectrometry is that this technology cannot be used to measure extremely large and heavily glycosylated proteins such as CA125. The Luminex LabMap technology permits simultaneous assay of multiple analytes $(>20)$ with small volumes of serum $(50 \mathrm{uL})$. This system uses polystyrene microspheres internally dyed with different ratios of two spectrally distinct fluorophores to create a family of up to 100 different spectrally addressed bead sets. Each of the bead sets is conjugated with a different capture antibody specific for a unique target protein. Fluorescent probes are linked to different antibodies that bind to distinct epitopes on each of the captured analytes to create sandwich assays. The doubly fluorescent beads are then analyzed by flow cytometry. Lokshin and colleagues have established and validated more than 50 Luminex assays that include CA125, MUC1, CA 153, CA 19-9, MMP2, MMP9, MCP1, IL-6, IL-8, IL13, VEGF, soluble EGFR, osteopontin, and kallikrein 10 [25,47]. Using the Luminex format, it was possible to achieve a sensitivity of $84 \%$ at a specificity of $95 \%$ with IL-6, IL-8, VEGF, EGF and CA125.

\subsection{Urine markers for early stage ovarian cancer}

Most ovarian cancer biomarkers have been measured in serum of patients with early stage disease, less attention has been given to potential biomarkers in the urine. Identification of urine markers could provide a more convenient and less invasive initial step in a two stage strategy. Urine proteins are generally small and therefore more thermodynamically stable [40]. Urine also tends to be less complex when compared to serum [106] which can be an advantage in proteomic analysis. Recently, eosinophil derived neurotoxin (EDN) and a fragment of osteopontin have been detected in the urine of patients with early stage ovarian cancer [106]. In earlier studies, Cole, et al, had reported elevation of human chorionic gonadotropin (hCG) free beta subunit and beta subunit core fragment in the urine of women with ovarian cancer [15]. The free beta subunit of hCG is a nicked form of the beta subunit that results in its disassociation from the alpha subunit and this form is removed from circulation at a much greater rate than intact hCG. The free beta subunit can be further degraded in the kidney and excreted into the urine as the beta-subunit core fragment. Our laboratory measured SMRP in the serum and urine of patients with invasive ovarian cancers and tumors of low malignant potential. Thresholds were set to include 95\% of SMRP values for sera and urines from healthy women. Urine values were considered: 1) as assayed; 2) normalized using the ratio of serum to urine creatinine; and 3 ) normalized using the Cockroft-Gault formula for glomerular filtration rate (GFR) which includes the age and weight of the patient. Interestingly, a greater fraction of patients with early stage disease was detected with the urine assay than with the serum assay for SMRP. Optimal sensitivity for early stage disease was obtained when data were normalized using the GFR. SMRP levels were elevated in urine from nearly $42 \%$ of patients with early stage ovarian cancer and $75 \%$ of patients with late stage ovarian cancer [3].

\section{Conclusions and future directions}

Early detection of ovarian cancer still holds great promise as an approach to reduce mortality from this disease. Within the next 5 years, results of the UKCTOCS trial in the United Kingdom should be available to test whether survival can be improved through use of sequential analysis of CA125 followed by TVS. If this trial is positive, there will still be a need to improve sensitivity of the initial phase through the use of multiple biomarkers. Currently, investigators from the NCI-funded Ovarian SPOREs are collaborating with each other and with the Early Detection Research Network and the PLCO to evaluate most of the markers in development listed above as well as the multiplex approach to detect preclinical disease among PLCO samples. These studies should provide guidance in prioritizing candidate biomarkers. Additional development of statistical methods will be required if multiple markers are to be followed over time to more precisely define the risk of ovarian cancer. Detection of sporadic disease and of hereditary disease may prove to be different problems, given the biological differences noted above. In the long run, further exploration of urine biomarkers may be desirable, considering the potential convenience of urinary assays in screening populations. 


\section{Acknowledgements}

Support is gratefully acknowledged from the Ovarian Cancer Research Fund, Golfers Against Cancer, the Mossy Foundation, the HERA Foundation, the Tracey Jo Wilson Foundation and NCI SPORE Grant No. 5 P50 CA08363907.

\section{References}

[1] American Cancer Society Statistics for 2006, http://www. cancer.org/docroot/STT/stt_0.asp.

[2] G.L. Andriole, D. Reding, R.B. Hayes, P.C. Prorok and J.K. Gohagan, The prostate, lung, colon, and ovarian (PLCO) cancer screening trial: Status and promise, Urol Oncol 22(4) (2004), 358-361.

[3] D. Badgwell, Z. Lu, H. Fritsche, E.N. Atkinson, E. Somers, J. Allard, R.G. Moore, K. Lu and R.C. Bast, Jr., Soluble mesothelin-related protein (SMRP) is elevated in the serum and urine of patients with early stage ovarian cancer, $14^{\text {th }}$ Annual SPORE Workshop Abstract \#018 (2006).

[4] K.A. Baggerly, S.R. Edmonson, J.S. Morris and K.R. Coombes, High-resolution serum proteomic patterns for ovarian cancer detection, Endocr Relat Cancer 11(4) (2004), 583-584; author reply 585-587.

[5] K.A. Baggerly, J.S. Morris, S.R. Edmonson and K.R. Coombes, Signal in noise: Evaluating reported reproducibility of serum proteomic tests for ovarian cancer, J Natl Cancer Inst 97(4) (2005), 307-309.

[6] R.C. Bast, Jr., Status of tumor markers in ovarian cancer screening, J Clin Oncol 21(10) (2003), 200-205.

[7] R.C. Bast, Jr., N. Urban, V. Shridhar, D. Smith, Z. Zhang, S. Skates, K. Lu, J. Liu, D. Fishman and G. Mills. Early detection of ovarian cancer: promise and reality, in: Biology of ovarian cancer, S. Stack and D. Fishman, eds, Dordrecht (The Netherlands): Kluwer Academic Publishers. 2002.

[8] R.C. Bast, Jr., D. Badgwell, Z. Lu, R. Marquez, D. Rosen, J. Liu, K.A. Baggerly, E.N. Atkinson, S. Skates, Z. Zhang, A. Lokshin, U. Menon, I. Jacobs and K. Lu, New tumor markers: CA125 and beyond, Int J Gynecol Cancer 15(3) (2005), 274-281.

[9] R.C. Bast, Jr., N. Urban, V. Shridhar, D. Smith, Z. Zhang, S. Skates, K. Lu, J. Liu, D. Fishman and G. Mills, Early detection of ovarian cancer: Promise and reality, Cancer Treat Res 107 (2002), 61-97.

[10] E. Bignotti, R.A. Tassi, S. Calza, A. Ravaggi, C. Romani, E. Rossi, M. Falchetti, F.E. Odicino, S. Pecorelli and A.D. Santin, Differential gene expression profiles between tumor biopsies and short-term primary cultures of ovarian serous carcinomas: Identification of novel molecular biomarkers for early diagnosis and therapy, Gynecol Oncol 103(2) (2006), 405-416.

[11] T.H. Bourne, S. Campbell, K.M. Reynolds, M.I. Whitehead, J. Hampson, P. Royston, T.J. Crayford and W.P. Collins, Screening for early familial ovarian cancer with transvaginal ultrasonography and colour blood flow imaging, $B M J$ 306(6884) (1993), 1025-1029.

[12] J. Boyd, Hereditary Ovarian Cancer: What we know, Gynecol Oncol 88 (2003), S8-S10.
[13] S.S. Buys, E. Partridge, M.H. Greene, P.C. Prorok, D. Reding, T.L. Riley, P. Hatge, R.M. Fagerstrom, L.R. Ragard, G. Izmirlian, M. Fouad, C.C. Johnson, J.K. Gohagan and PCLO Project Team. Ovarian cancer screening in the Prostate, Lung, Colorectal and Ovarian (PLCO) cancer screening trial: findings from the initial screen of a randomized trial, Am J Obstet Gynecol 193(6) (2005), 2183-2184.

[14] S. Campbell, V. Bhan, P. Royston, M.I. Whitehead and W.P. Collins, Transabdominal ultrasound screening for early ovarian cancer, BMJ 299(6712) (1989), 1363-1367.

[15] L.A. Cole and J.H. Nam, Urinary gonadotropin fragment (UGF) measurements in the diagnosis and management of ovarian cancer, Yale J Biol Med 62(4) (1989), 367-378.

[16] T.P. Conrads, V.A. Fusaro, S. Ross, D. Johann, V. Rajapakse, B.A. Hitt, S.M. Steinberg, E.C. Kohn, D.A. Fishman, G. Whitely, J.C. Barrett, L.A. Liotta, E.F. Petricoin, 3rd and T.D. Veenstra, High-resolution serum proteomic features for ovarian cancer detection, Endocr Relat Cancer 11(2) (2004), $163-178$.

[17] T.P. Conrads, M. Zhou, E.F. Petricoin, 3rd, L. Liotta and T.D. Veenstra, Cancer diagnosis using proteomic patterns, Expert Rev Mol Diagn 3(4) (2003), 411-420.

[18] E.P. Diamandis, A. Okui, S. Mitsui, L.Y. Luo, A. Soosaipillai, L. Grass, T. Nakamura, D.J. Howarth and N. Yamaguchi, Human kallikrein 11: A new biomarker of prostate and ovarian carcinoma, Cancer Res 62(1) (2002), 295-300.

[19] E.P. Diamandis, G.M. Yousef, A.R. Soosaipillai and P. Bunting, Human kallikrein 6 (zyme/protease m/neurosin): A new serum biomarker of ovarian carcinoma, Clin Biochem 33(7) (2000), 579-583.

[20] I. Dimova, B. Zaharieva, S. Raitcheva, R. Dimitrov, N. Doganov and D. Toncheva, Tissue microarray analysis of egfr and erbb2 copy number changes in ovarian tumors, Int J Gynecol Cancer 16(1) (2006), 145-151.

[21] L. Dubeau, The cell of origin of ovarian epithelial tumors and the ovarian surface epithelium dogma: Does the emperor have no clothes? Gynecol Oncol 72(3) (1999), 437-442.

[22] X. Fang, S. Yu, R.C. Bast, S. Liu, H.J. Xu, S.X. Hu, R. LaPushin, F.X. Claret, B.B. Aggarwal, Y. Lu and G.B. Mills, Mechanisms for lysophosphatidic acid-induced cytokine production in ovarian cancer cells, J Biol Chem 279(10) (2004), 9653-9661.

[23] A. Finch, M. Beiner, J. Lubinski, H. T. Lynch, P. Moller, B. Rosen, J. Murphy, P. Ghadirian, E. Friedman, W. D. Foulkes, C. Kim-Sing, T. Wagner, N. Tung, F. Couch, D. StoppaLyonnet, P. Ainsworth, M. Daly, B. Pasini, R. GershoniBaruch, C. Eng, O.I. Olopade, J. McLennan, B. Karlan, J. Weitzel, P. Sun and S.A. Narod, Salpingo-oophorectomy and the risk of ovarian, fallopian tube, and peritoneal cancers in women with a brca1 or brca2 mutation, Jama 296(2) (2006), 185-192.

[24] M.A. Gillette, D.R. Mani and S.A. Carr, Place of pattern in proteomic biomarker discovery, J Proteome Res 4(4) (2005), 1143-1154.

[25] E. Gorelik, D.P. Landsittel, A.M. Marrangoni, F. Modugno, L. Velikokhatnaya, M.T. Winans, W.L. Bigbee, R.B. Herberman and A.E. Lokshin, Multiplexed immunobead-based cytokine profiling for early detection of ovarian cancer, Cancer Epidemiol Biomarkers Prev 14(4) (2005), 981-987.

[26] T. Goto, M. Takano, M. Sakamoto, A. Kondo, J. Hirata, T. Kita, H. Tsuda, Y. Tenjin and Y. Kikuchi, Gene expression profiles with cDNA microarray reveal rhogdi as a predictive marker for paclitaxel resistance in ovarian cancers, Oncol Rep 15(5) (2006), 1265-1271. 
[27] C. Haaften-Day, Y. Shen, X. Fengii, Y. Yu, A. Berchuck, L.J. Havrilesky, H.W. A. de Bruijn, A.G.J. van der Zee, R.C. Bast and N.F. Hacker, OVX1, Macrophage-Colony Stimulating Factor, and CA-125-II as Tumor Markers for Epithelial Ovarian Carcinoma, Cancer 92(11) (2001), 2837-2844.

[28] R.B. Hayes, D. Reding, W. Kopp, A.F. Subar, N. Bhat, N. Rothman, N. Caporaso, R.G. Ziegler, C.C. Johnson, J.L. Weissfeld, R.N. Hoover, P. Hartge, C. Palace and J.K. Gohagan, Etiologic and early marker studies in the prostate, lung, colorectal and ovarian (plco) cancer screening trial, Control Clin Trials 21(6) (2000), 349S-355S.

[29] I. Hellstrom, J. Raycraft, M. Hayden-Ledbetter, J.A. Ledbetter, M. Schummer, M. McIntosh, C. Drescher, N. Urban and K.E. Hellstrom, The he4 (wfdc2) protein is a biomarker for ovarian carcinoma, Cancer Res 63(13) (2003), 3695-3700.

[30] E.V. Hogdall, L. Christensen, S.K. Kjaer, J. Blaakaer, A. Kjaerbye-Thygesen, S. Gayther, I.J. Jacobs and C.K. Hogdall, CA125 expression pattern, prognosis and correlation with serum ca125 in ovarian tumor patients from the danish "Malova" Ovarian cancer study, Gynecol Oncol (2006).

[31] C.D. Hough, C.A. Sherman-Baust, E.S. Pizer, F.J. Montz, D.D. Im, N.B. Rosenshein, K.R. Cho, G.J. Riggins and P.J. Morin, Large-scale serial analysis of gene expression reveals genes differentially expressed in ovarian cancer, Cancer Res 60(22) (2000), 6281-6287.

[32] I. Jacobs, Screening for ovarian cancer by CA-125 measurement, Lancet 1 (8590) (1988), 889.

[33] I. Jacobs and D. Oram, Screening for ovarian cancer, Biomed Pharmacother 42(9) (1988), 589-596.

[34] I. Jacobs, I. Stabile, J. Bridges, P. Kemsley, C. Reynolds, J. Grudzinskas and D. Oram, Multimodal approach to screening for ovarian cancer, Lancet 1(8580) (1988), 268-271.

[35] I.J. Jacobs, D.H. Oram and R.C. Bast, Jr., Strategies for improving the specificity of screening for ovarian cancer with tumor-associated antigens CA125, CA15-3, and TAG72.3, Obstet Gynecol 80(3) (1992), Pt 1, 396-399.

[36] I.J. Jacobs, S.J. Skates, N. MacDonald, U. Menon, A.N. Rosenthal, A.P. Davies, R. Woolas, A.R. Jeyarajah, K. Sibley, D.G. Lowe and D.H. Oram, Screening for ovarian cancer: A pilot randomised controlled trial, Lancet 353(9160) (1999), $1207-1210$.

[37] J.H. Kim, S.J. Skates, T. Uede, K.K. Wong, J.O. Schorge, C.M. Feltmate, R.S. Berkowitz, D.W. Cramer and S.C. Mok, Osteopontin as a potential diagnostic biomarker for ovarian cancer, Jama 287(13) (2002), 1671-1679.

[38] F. Kong, C. Nicole White, X. Xiao, Y. Feng, C. Xu, D. He, $\mathrm{Z}$. Zhang and $\mathrm{Y}$. Yu, Using proteomic approaches to identify new biomarkers for detection and monitoring of ovarian cancer, Gynecol Oncol 100(2) (2006), 247-253.

[39] K.R. Kozak, F. Su, J.P. Whitelegge, K. Faull, S. Reddy and R. Farias-Eisner, Characterization of serum biomarkers for detection of early stage ovarian cancer, Proteomics 5(17) (2005), 4589-4596.

[40] S. Kumar, C.J. Tsai and R. Nussinov, Temperature range of thermodynamic stability for the native state of reversible two-state proteins, Biochemistry 42(17) (2003), 4864-4873.

[41] J.M. Lancaster, H.K. Dressman, J.P. Clarke, R.A. Sayer, M.A. Martino, J.M. Cragun, A.H. Henriott, J. Gray, R. Sutphen, A. Elahi, R.S. Whitaker, M. West, J.R. Marks, J.R. Nevins and A. Berchuck, Identification of genes associated with ovarian cancer metastasis using microarray expression analysis, Int J Gynecol Cancer 16(5) (2006), 1733-1745.

[42] C. Le Page, V. Ouellet, J. Madore, F. Ren, T.J. Hudson, P.N. Tonin, D.M. Provencher and A.M. Mes-Masson, Gene expression profiling of primary cultures of ovarian epithelial cells identifies novel molecular classifiers of ovarian cancer, Br J Cancer 94(3) (2006), 436-445.

[43] Y. Lee, A. Miron, R. Drapkin, M.R. Nucci, F. Medeiros, A. Saleemuddin, J. Garber, C. Birch, H. Mou, R.W. Gordon, D.W. Cramer, F.D. McKeon and C.P. Crum, A candidate precursor to serous carcinoma that originates in the distal fallopian tube, J Pathol 211(1) (2007), 26-35.

[44] S. L'Esperance, I. Popa, M. Bachvarova, M. Plante, N. Patten, L. Wu, B. Tetu and D. Bachvarov, Gene expression profiling of paired ovarian tumors obtained prior to and following adjuvant chemotherapy: Molecular signatures of chemoresistant tumors, Int J Oncol 29(1) (2006), 5-24.

[45] S. Li, H. Han, E. Resnik, M.L. Carcangiu, P.E. Schwartz and T.L. Yang-Feng, Advanced ovarian carcinoma: Molecular evidence of unifocal origin, Gynecol Oncol 51(1) (1993), 21-25.

[46] Y.W. Lin, C.Y. Lin, H.C. Lai, J.Y. Chiou, C.C. Chang, M.H. Yu and T.Y. Chu, Plasma proteomic pattern as biomarkers for ovarian cancer, Int J Gynecol Cancer 16(1) (2006), 139-146.

[47] A.E. Lokshin, M. Winans, D. Landsittel, A.M. Marrangoni, L. Velikokhatnaya, F. Modugno, B.M. Nolen and E. Gorelik, Circulating il-8 and anti-il-8 autoantibody in patients with ovarian cancer, Gynecol Oncol 102(2) (2006), 244-251.

[48] K.H. Lu, A.P. Patterson, L. Wang, R.T. Marquez, E.N. Atkinson, K.A. Baggerly, L.R. Ramoth, D.G. Rosen, J. Liu, I. Hellstrom, D. Smith, L. Hartmann, D. Fishman, A. Berchuck, R. Schmandt, R. Whitaker, D.M. Gershenson, G.B. Mills and R.C. Bast, Jr., Selection of potential markers for epithelial ovarian cancer with gene expression arrays and recursive descent partition analysis, Clin Cancer Res 10(10) (2004), 3291-3300.

[49] L.Y. Luo, P. Bunting, A. Scorilas and E.P. Diamandis, Human kallikrein 10: A novel tumor marker for ovarian carcinoma? Clin Chim Acta 306(1-2) (2001), 111-118.

[50] L. Manenti, P. Paganoni, I. Floriani, F. Landoni, V. Torri, A. Buda, G. Taraboletti, R. Labianca, D. Belotti and R. Giavazzi, Expression levels of vascular endothelial growth factor, matrix metalloproteinases 2 and 9 and tissue inhibitor of metalloproteinases 1 and 2 in the plasma of patients with ovarian carcinoma, Eur J Cancer 39(13) (2003), 1948-1956.

[51] R.T. Marquez, K.A. Baggerly, A.P. Patterson, J. Liu, R. Broaddus, M. Frumovitz, E.N. Atkinson, D.I. Smith, L. Hartmann, D. Fishman, A. Berchuck, R. Whitaker, D.M. Gershenson, G.B. Mills, R.C. Bast, Jr. and K.H. Lu, Patterns of gene expression in different histotypes of epithelial ovarian cancer correlate with those in normal fallopian tube, endometrium, and colon, Clin Cancer Res 11(17) (2005), 6116-6126.

[52] D. Mayr, V. Kanitz, B. Anderegg, B. Luthardt, J. Engel, U. Lohrs, G. Amann and J. Diebold, Analysis of gene amplification and prognostic markers in ovarian cancer using comparative genomic hybridization for microarrays and immunohistochemical analysis for tissue microarrays, Am J Clin Pathol 126(1) (2006), 101-109.

[53] M.W. McIntosh, C. Drescher, B. Karlan, N. Scholler, N. Urban, K.E. Hellstrom and I. Hellstrom, Combining CA125 and SMR serum markers for diagnosis and early detection of ovarian carcinoma, Gynecol Oncol 95(1) (2004), 9-15.

[54] I. Meinhold-Heerlein, D. Bauerschlag, F. Hilpert, P. Dimitrov, L.M. Sapinoso, M. Orlowska-Volk, T. Bauknecht, T.W. Park, W. Jonat, A. Jacobsen, J. Sehouli, J. Luttges, M. Krajewski, S. Krajewski, J. C. Reed, N. Arnold and G.M. Hampton,Molecular and prognostic distinction be- 
tween serous ovarian carcinomas of varying grade and malignant potential, Oncogene 24(6) (2005), 1053-1065.

[55] U. Menon, S.J. Skates, S. Lewis, A.N. Rosenthal, B. Rufford, K. Sibley, N. Macdonald, A. Dawnay, A. Jeyarajah, R.C. Bast, Jr., D. Oram and I.J. Jacobs, Prospective study using the risk of ovarian cancer algorithm to screen for ovarian cancer, J Clin Oncol 23(31) (2005), 7919-7926.

[56] U. Menon, A. Talaat, A.R. Jeyarajah, A.N. Rosenthal, N.D. MacDonald, S.J. Skates, K. Sibley, D.H. Oram and I.J. Jacobs, Ultrasound assessment of ovarian cancer risk in postmenopausal women with CA125 elevation, $\mathrm{Br} J$ Cancer 80(10) (1999), 1644-1647.

[57] G.B. Mills, C. May, M. McGill, C.M. Roifman and A. Mellors, A putative new growth factor in ascitic fluid from ovarian cancer patients: Identification, characterization, and mechanism of action, Cancer Res 48(5) (1988), 1066-1071.

[58] C.H. Mok, S.W. Tsao, R.C. Knapp, P.M. Fishbaugh and C.C. Lau, Unifocal origin of advanced human epithelial ovarian cancers, Cancer Res 52(18) (1992), 5119-5122.

[59] S.C. Mok, J. Chao, S. Skates, K. Wong, G.K. Yiu, M.G. Muto, R.S. Berkowitz and D.W. Cramer, Prostasin, a potential serum marker for ovarian cancer: Identification through microarray technology, J Natl Cancer Inst 93(19) (2001), 1458-1464.

[60] L.E. Moore, E.T. Fung, M. McGuire, C.C. Rabkin, A. Molinaro, Z. Wang, F. Zhang, J. Wang, C. Yip, X.Y. Meng and R.M. Pfeiffer, Evaluation of Apolipoprotein A1 and Posttranslationally Modified Forms of Transthyretin as Biomarkers for Ovarian Cancer Detection in an Independent Study Population, Cancer Epidemiol Biomarkers Prev 15(9) (2006), 1641-1646.

[61] R.G. Moore, A.K. Brown, C.M. Miller, D. Badgwell, Z. Lu, T. Verch, K.H. Lu, J.W. Allard, C.O. Granai and R.C. Bast, Jr., A novel multiple biomarker assay for the detection of ovarian carcinoma,J Clin Oncol 24(18S) (2006), 5023.

[62] G. Mor, I. Visintin, Y. Lai, H. Zhao, P. Schwartz, T. Rutherford, L. Yue, P. Bray-Ward and D.C. Ward, Serum protein markers for early detection of ovarian cancer, Proc Natl Acad Sci USA 102(21) (2005), 7677-7682.

[63] M. Moscova, D.J. Marsh and R.C. Baxter, Protein chip discovery of secreted proteins regulated by the phosphatidylinositol 3-kinase pathway in ovarian cancer cell lines, Cancer Res 66(3) (2006), 1376-1383.

[64] R. Moszynski, D. Szpurek, A. Smolen and S. Sajdak, Comparison of diagnostic usefulness of predictive models in preliminary differentiation of adnexal masses, Int J Gynecol Cancer 16(1) (2006), 45-51.

[65] M. Nakae, I. Iwamoto, T. Fujino, Y. Maehata, S. Togami, M. Yoshinaga and T. Douchi, Preoperative plasma osteopontin level as a biomarker complementary to carbohydrate antigen 125 in predicting ovarian cancer, J Obstet Gynaecol Res 32(3) (2006), 309-314.

[66] M. Nap, A. Vitali, K. Nustad, R.C. Bast, Jr., T.J. O’Brien, O. Nilsson, P. Seguin, M.R. Suresh, O.P. Bormer, T. Saga, H.W. de Bruijn, S. Nozawa, F.T. Kreutz, D. Jette, H. Sakahara, M. Gadnell, K. Endo, E.H. Barlow, D. Warren, E. Paus, S. Hammarstrom, P. Kenemans and J. Hilgers, Immunohistochemical characterization of 22 monoclonal antibodies against the CA125 antigen: 2nd report from the isobm td-1 workshop, Tumour Biol 17(6) (1996), 325-331.

[67] T.J. O'Brien, L.M. Raymond, G.A. Bannon, D.H. Ford, H. Hardardottir, F.C. Miller and J.G. Quirk, Jr., New monoclonal antibodies identify the glycoprotein carrying the CA125 epitope, Am J Obstet Gynecol 165(6) (1991), Pt 1, 18571864.

[68] R.I. Olivier, M. van Beurden and T.V.L.J. Van, The role of gene expression profiling in the clinical management of ovarian cancer, Eur J Cancer 42(17) (2006), 2930-2938.

[69] C. Peters-Engl, M. Medl, E. Ogris and S. Leodolter, Tumorassociated trypsin inhibitor (tati) and cancer antigen 125 (CA125) in patients with epithelial ovarian cancer, Anticancer Res 15(6B) (1995), 2727-2730.

[70] E.F. Petricoin, A.M. Ardekani, B.A. Hitt, P.J. Levine, V.A. Fusaro, S.M. Steinberg, G.B. Mills, C. Simone, D.A. Fishman, E.C. Kohn and L.A. Liotta, Use of proteomic patterns in serum to identify ovarian cancer, Lancet 359(9306) (2002), 572-577.

[71] N. Rifai, M.A. Gillette and S.A. Carr, Protein biomarker discovery and validation: The long and uncertain path to clinical utility, Nat Biotechnol 24(8) (2006), 971-983.

[72] D.G. Rosen, L. Wang, J.N. Atkinson, Y. Yu, K.H. Lu, E.P. Diamandis, I. Hellstrom, S.C. Mok, J. Liu and R.C. Bast, Jr., Potential markers that complement expression of CA 125 in epithelial ovarian cancer, Gynecol Oncol 99(2) (2005), 267-277.

[73] A. Rosenthal and I. Jacobs, Familial ovarian cancer screening, Best Pract Res Clin Obstet Gynaecol 20(2) (2006), 321338.

[74] A.N. Rosenthal and I.J. Jacobs, The role of CA125 in screening for ovarian cancer, Int J Biol Markers 13(4) (1998), 216220.

[75] A.N. Rosenthal, U. Menon and I.J. Jacobs, Screening for ovarian cancer, Clin Obstet Gynecol 49(3) (2006), 433-447.

[76] S. Salceda, T. Tang, M. Kmet, A. Munteanu, M. Ghosh, R. Macina, W. Liu, G. Pilkington and J. Papkoff, The immunomodulatory protein B7-H4 is overexpressed in breast and ovarian cancers and promotes epithelial cell transformation, Exp Cell Res 306(1) (2005), 128-141.

[77] A.H. Sato, G.L. Anderson, N. Urban and M.W. McIntosh, Comparing adaptive and non-adaptive algorithms for cancer early detection with novel biomarkers, Cancer Biomark 2(34) (2006), 151-162.

[78] S. Sato, Y. Yokoyama, T. Sakamoto, M. Futagami and Y. Saito, Usefulness of mass screening for ovarian carcinoma using transvaginal ultrasonography, Cancer 89(3) (2000), 582-588.

[79] N. Scholler, N. Fu, Y. Yang, Z. Ye, G.E. Goodman, K.E. Hellstrom and I. Hellstrom, Soluble member(s) of the mesothelin/megakaryocyte potentiating factor family are detectable in sera from patients with ovarian carcinoma, Proc Natl Acad Sci USA 96(20) (1999), 11531-11536.

[80] M. Schummer, W.V. Ng, R.E. Bumgarner, P.S. Nelson, B. Schummer, D.W. Bednarski, L. Hassell, R.L. Baldwin, B.Y. Karlan and L. Hood, Comparative hybridization of an array of 21,500 ovarian cDNAs for the discovery of genes overexpressed in ovarian carcinomas, Gene 238(2) (1999), 375385.

[81] D.R. Schwartz, S.L. Kardia, K.A. Shedden, R. Kuick, G. Michailidis, J.M. Taylor, D.E. Misek, R. Wu, Y. Zhai, D.M. Darrah, H. Reed, L.H. Ellenson, T.J. Giordano, E.R. Fearon, S.M. Hanash and K.R. Cho, Gene expression in ovarian cancer reflects both morphology and biological behavior, distinguishing clear cell from other poor-prognosis ovarian carcinomas, Cancer Res 62(16) (2002), 4722-4729.

[82] D.R. Schwartz, R. Wu, S.L. Kardia, A.M. Levin, C.C. Huang, K.A. Shedden, R. Kuick, D.E. Misek, S.M. Hanash, J.M. Taylor, H. Reed, N. Hendrix, Y. Zhai, E.R. Fearon and K.R. 
Cho, Novel candidate targets of beta-catenin/t-cell factor signaling identified by gene expression profiling of ovarian endometrioid adenocarcinomas, Cancer Res 63(11) (2003), 2913-2922.

[83] V. Shridhar, J. Lee, A. Pandita, S. Iturria, R. Avula, J. Staub, M. Morrissey, E. Calhoun, A. Sen, K. Kalli, G. Keeney, P. Roche, W. Cliby, K. Lu, R. Schmandt, G.B. Mills, R.C. Bast, Jr., C.D. James, F.J. Couch, L.C. Hartmann, J. Lillie and D.I. Smith, Genetic analysis of early- versus late-stage ovarian tumors, Cancer Res 61(15) (2001), 5895-5904.

[84] H.S. Shvartsman, K.H. Lu, J. Lee, J. Lillie, M.T. Deavers, S. Clifford, J.K. Wolf, G.B. Mills, R.C. Bast, Jr., D.M. Gershenson and R. Schmandt, Overexpression of kallikrein 10 in epithelial ovarian carcinomas, Gynecol Oncol 90(1) (2003), 44-50.

[85] I. Simon, S. Zhuo, L. Corral, E.P. Diamandis, M.J. Sarno, R.L. Wolfert and N.W. Kim, B7-H4 is a novel membranebound protein and a candidate serum and tissue biomarker for ovarian cancer, Cancer Res 66(3) (2006), 1570-1575.

[86] K. Sjovall, B. Nilsson and N. Einhorn, The significance of serum CA125 elevation in malignant and nonmalignant diseases, Gynecol Oncol 85(1) (2002), 175-178.

[87] S. Skates, R. Troiano and R.C. Knapp, Longitudinal CA125 detection of sporadic papillary serous carcinoma of the peritoneum, Int J Gynecol Cancer 13(5) (2003), 693-696.

[88] S.J. Skates, N. Horick, Y. Yu, F.J. Xu, A. Berchuck, K.J. Havrilesky, H.W. de Bruijn, A.G. van der Zee, R.P. Woolas, I.J. Jacobs, Z. Zhang and R.C. Bast, Jr., Preoperative sensitivity and specificity for early-stage ovarian cancer when combining cancer antigen CA125II, CA15-3, CA72-4, and macrophage colony-stimulating factor using mixtures of multivariate normal distributions, J Clin Oncol 22(20) (2004), 4059-4066.

[89] S.J. Skates, U. Menon, N. MacDonald, A.N. Rosenthal, D.H. Oram, R.C. Knapp and I.J. Jacobs, Calculation of the risk of ovarian cancer from serial CA125 values for preclinical detection in postmenopausal women, J Clin Oncol 21(10) (2003), 206-210.

[90] S.J. Skates and D.E. Singer, Quantifying the potential benefit of CA125 screening for ovarian cancer, J Clin Epidemiol 44(4-5) (1991), 365-380.

[91] S.J. Skates, F.J. Xu, Y.H. Yu, K. Sjovall, N. Einhorn, Y. Chang, R.C. Bast, Jr. and R.C. Knapp, Toward an optimal algorithm for ovarian cancer screening with longitudinal tumor markers, Cancer 76(10) (1995), 2004-2010.

[92] L.H. Smith, Early clinical detection of ovarian cancer: A review of the evidence, Expert Rev Anticancer Ther 6(7) (2006), 1045-1052.

[93] L.H. Smith, C.R. Morris, S. Yasmeen, A. Parikh-Patel, R.D. Cress and P.S. Romano, Ovarian cancer: Can we make the clinical diagnosis earlier? Cancer 104(7) (2005), 13981407.

[94] R. Sutphen, Y. Xu, G.D. Wilbanks, J. Fiorica, E.C. Grendys, Jr., J.P. LaPolla, H. Arango, M.S. Hoffman, M. Martino, K. Wakeley, D. Griffin, R.W. Blanco, A.B. Cantor, Y.J. Xiao and J.P. Krischer, Lysophospholipids are potential biomarkers of ovarian cancer, Cancer Epidemiol Biomarkers Prev 13(7) (2004), 1185-1191.

[95] D. Szpurek, R. Moszynski, A. Smolen and S. Sajdak, Artificial neural network computer prediction of ovarian malignancy in women with adnexal masses, Int J Gynaecol Obstet 89(2) (2005), 108-113.

[96] B. Tringler, W. Liu, L. Corral, K.C. Torkko, T. Enomoto, S. Davidson, M.S. Lucia, D.E. Heinz, J. Papkoff and K.R.
Shroyer, B7-H4 overexpression in ovarian tumors, Gynecol Oncol 100(1) (2006), 44-52.

[97] N. Urban, Ovarian Cancer Risk Assessment and the Potential for Early Detection, Gynecol Oncol 88 (2003), S75-S79.

[98] C. van Haaften-Day, Y. Shen, F. Xu, Y. Yu, A. Berchuck, L.J. Havrilesky, H.W. de Bruijn, A.G. van der Zee, R.C. Bast, Jr. and N.F. Hacker, Ovx1, macrophage-colony stimulating factor, and CA125II as tumor markers for epithelial ovarian carcinoma: A critical appraisal, Cancer 92(10) (2001), 2837-2844.

[99] J.R. van Nagell, Jr., P.D. DePriest, M.B. Reedy, H.H. Gallion, F.R. Ueland, E.J. Pavlik and R.J. Kryscio, The efficacy of transvaginal sonographic screening in asymptomatic women at risk for ovarian cancer, Gynecol Oncol 77(3) (2000), 350356.

[100] K.K. Wong, R.S. Cheng and S.C. Mok, Identification of differentially expressed genes from ovarian cancer cells by MICROMAX cDNA microarray system, Biotechniques 30 (2001), 670-675.

[101] F.J. Xu, S. Ramakrishnan, L. Daly, J.T. Soper, A. Berchuck, D. Clarke-Pearson and R.C. Bast, Jr., Increased serum levels of macrophage colony-stimulating factor in ovarian cancer, Am J Obstet Gynecol 165(5) (1991), Pt 1, 1356-1362.

[102] F.J. Xu, Y.H. Yu, L. Daly, K. DeSombre, L. Anselmino, G.M. Hass, A. Berchuck, J.T. Soper, D.L. Clarke-Pearson, C. Boyer et al., Ovx1 radioimmunoassay complements CA125 for predicting the presence of residual ovarian carcinoma at second-look surgical surveillance procedures, J Clin Oncol 11(8) (1993), 1506-1510.

[103] Y. Xu, D.C. Gaudette, J.D. Boynton, A. Frankel, X.J. Fang, A. Sharma, J. Hurteau, G. Casey, A. Goodbody, A. Mellors et al., Characterization of an ovarian cancer activating factor in ascites from ovarian cancer patients, Clin Cancer Res 1(10) (1995), 1223-1232.

[104] Y. Xu, Z. Shen, D.W. Wiper, M. Wu, R.E. Morton, P. Elson, A.W. Kennedy, J. Belinson, M. Markman and G. Casey, Lysophosphatidic acid as a potential biomarker for ovarian and other gynecologic cancers, Jama 280(8) (1998), 719723.

[105] S. Yamamoto, I. Konishi, M. Mandai, H. Kuroda, T. Komatsu, K. Nanbu, H. Sakahara and T. Mori, Expression of vascular endothelial growth factor (VEGF) in epithelial ovarian neoplasms: Correlation with clinicopathology and patient survival, and analysis of serum vegf levels, Br J Cancer 76(9) (1997), 1221-1227.

[106] B. Ye, S. Skates, S.C. Mok, N.K. Horick, H.F. Rosenberg, A. Vitonis, D. Edwards, P. Sluss, W.K. Han, R.S. Berkowitz and D.W. Cramer, Proteomic-based discovery and characterization of glycosylated eosinophil-derived neurotoxin and cooh-terminal osteopontin fragments for ovarian cancer in urine, Clin Cancer Res 12(2) (2006), 432-441.

[107] B.W. Yin, A. Dnistrian and K.O. Lloyd, Ovarian cancer antigen CA125 is encoded by the muc16 mucin gene, Int J Cancer 98(5) (2002), 737-740.

[108] G.M. Yousef and E.P. Diamandis, Expanded human tissue kallikrein family-a novel panel of cancer biomarkers, Tumour Biol 23(3) (2002), 185-192.

[109] G.M. Yousef and E.P. Diamandis, Tissue kallikreins: New players in normal and abnormal cell growth? Thromb Haemost 90(1) (2003), 7-16.

[110] W. Yu, B. Wu, N. Lin, K. Stone, K. Williams and H. Zhao, Detecting and aligning peaks in mass spectrometry data with applications to MALDI, Comput Biol Chem 30(1) (2006), 27-38. 
[111] H. Zhang, B. Kong, X. Qu, L. Jia, B. Deng and Q. Yang, Biomarker discovery for ovarian cancer using SELDI-TOFMS, Gynecol Oncol 102(1) (2006), 61-66.

[112] L. Zhang, J. Huang, N. Yang, S. Liang, A. Barchetti, A. Giannakakis, M.G. Cadungog, A. O'Brien-Jenkins, M. Massobrio, K.F. Roby, D. Katsaros, P. Gimotty, R. Butzow, B.L. Weber and G. Coukos, Integrative genomic analysis of protein kinase c (PKC) family identifies PKCiota as a biomarker and potential oncogene in ovarian carcinoma, Cancer Res 66(9) (2006), 4627-4635.

[113] Z. Zhang, S.D. Barnhill, H. Zhang, F. Xu, Y. Yu, I. Jacobs, R.P. Woolas, A. Berchuck, K.R. Madyastha and R.C. Bast,
Jr., Combination of multiple serum markers using an artificial neural network to improve specificity in discriminating malignant from benign pelvic masses, Gynecol Oncol 73(1) (1999), 56-61.

[114] Z. Zhang, R.C. Bast, Jr., Y. Yu, J. Li, L.J. Sokoll, A.J. Rai, J.M. Rosenzweig, B. Cameron, Y.Y. Wang, X.Y. Meng, A. Berchuck, C. Van Haaften-Day, N.F. Hacker, H.W. de Bruijn, A.G. van der Zee, I.J. Jacobs, E.T. Fung and D.W. Chan, Three biomarkers identified from serum proteomic analysis for the detection of early stage ovarian cancer, Cancer Res 64(16) (2004), 5882-5890. 